\title{
Low-frequency electromagnetic response function for strong-coupling superconductors*
}

\author{
P. D. Scholten, J. D. Lejeune, ${ }^{\dagger}$ W. M. Saslow, and D. G. Naugle \\ Department of Physics, Texas A\&M University, College Station, Texas 77843
}

(Received 1 March 1977)

\begin{abstract}
A simple method for calculating the low-frequency electromagnetic response function from tunneling derived $\alpha^{2}(\omega) F(\omega)$ has been developed and applied to $\mathrm{Pb}, \mathrm{Pb}_{0.90} \mathrm{Bi}_{0.10}$, amorphous $\mathrm{Bi}$, amorphous $\mathrm{Pb}_{0.45} \mathrm{Bi}_{0.55}$, and amorphous $\mathrm{Sn}_{0.90} \mathrm{Cu}_{0.10}$. The results have been compared with previous calculations and experiments. A correction to the calculation of the $T=0$ values given by Kerchner and Ginsberg is given. Although the calculations agree with experiment for crystalline $\mathrm{Pb}$, the calculated temperature dependence is much stronger near $T_{c}$ than that inferred from kinetic inductance measurements for amorphous Bi.
\end{abstract}

\section{INTRODUCTION}

The influence of strong electron-phonon coupling on the electromagnetic properties of superconductors is well known. For example, the frequency dependence of the electrical conductivity in strongcoupling superconductors has been observed by both infrared ${ }^{1,2}$ and microwave ${ }^{3}$ techniques. Also, the magnitude and temperature dependence of the low-frequency conductivity for $\mathrm{Pb}$ have been inferred from measurements of magnetic field attenuation, ${ }^{4}$ while kinetic inductance ${ }^{5}$ measurements have been used to study this temperature dependence for amorphous superconductors. Closely related to the electrical conductivity of the superconductor is the critical current of a Josephson junction, and this has been measured for $\mathrm{Pb}^{6}$

The theory of the electromagnetic properties of strong-coupling superconductors was originally developed by $\mathrm{Nam}^{7,8}$ and a correction for higher frequencies, $\hbar \omega>2 \Delta_{0}$, was noted by Swihart and Shaw. ${ }^{9}$ Using this theory, low-temperature values of the low-frequency electrical conductivity $\sigma(\omega \rightarrow 0, T=0)$, or equivalently of the electromagnetic response function $I(\omega=0, \vec{R}=0, T=0)$, have been calculated for many materials ${ }^{10-12}$; however, the results were not always in agreement.

In this paper detailed calculations of the response functions for several strong-coupling superconductors are presented. Solutions of the nonlinear Eliashberg equations for the gap function $\Delta\left(i \omega_{n}\right)$ on the imaginary axis using the tunneling derived $\alpha^{2}(\omega) F(\omega)$ is used with Nam's theory to calculate $I(0,0, T)$. As a check of the calculation procedures, $I(0,0,0)$ has also been calculated by three additional methods using the same tunneling data for input: (i) the direct calculation by Harris ${ }^{13}$ of the imaginary part of the conductivity, $\sigma_{2}(\omega)$, as a function of frequency and then taking the limit of $\omega \mathrm{O}_{2}(\omega)$ as $\omega \rightarrow 0$, (ii) the Kerchner-Ginsberg ${ }^{11}$ expression for $\lim _{\omega \rightarrow 0}\left[\omega \sigma_{2}(\omega)\right]$, and (iii) calcula- tion with Nam's theory using $\Delta\left(i \omega_{n}\right)$ determined by a transformation of the real frequency gap function $\Delta(\omega)$ obtained during the inversion of tunneling data to the imaginary frequency function $\Delta\left(i \omega_{n}\right)$ using Cauchy's theorem. Note that a correction to the Kerchner-Ginsberg expression at $T=0$ has been found and there is now excellent agreement between the values of $I(0,0,0)$ computed by the four different methods.

In Sec. II the methods for calculating the response function are described in detail. In particular, the Eliashberg method with $\Delta\left(i \omega_{n}\right)$ provides a very fast computational method for calculating $I(0,0, T)$ at a large number of temperatures. In Sec. III the results of the calculations are presented and compared with experimental data.

\section{CALCULATION OF $I(0,0, T)$}

\section{A. General formulation}

The electromagnetic response of a superconductor to an electromagnetic field may be written

$$
\begin{aligned}
\overrightarrow{\mathrm{j}}(\overrightarrow{\mathrm{r}}, \omega)= & \frac{e^{2} N(0) v_{F}}{2 \pi^{2} \hbar c} \\
& \times \int \frac{\overrightarrow{\mathbf{R}}\left[\overrightarrow{\mathbf{R}} \cdot \overrightarrow{\mathrm{A}}_{\omega}\left(\overrightarrow{\mathbf{r}}^{\prime}\right)\right]}{R^{4}} I(\omega, \overrightarrow{\mathrm{R}}, T) d \overrightarrow{\mathrm{r}}^{\prime},
\end{aligned}
$$

where $N(0)$ and $v_{F}$ are the band density of states and Fermi velocity (not the renormalized values), $\overrightarrow{\mathrm{R}}=\overrightarrow{\mathrm{r}}^{\prime}-\overrightarrow{\mathrm{r}}$, and $I(\omega, \overrightarrow{\mathrm{R}}, T)$ is the electromagnetic response function, which may be approximated by

$$
I(0, R, T)=I(0,0, T) e^{-R / \xi(T, l)}
$$

with

$$
\xi^{-1}(T, l)=\xi_{0}^{-1}(T)+(\delta l)^{-1},
$$

where $\xi_{0}(T)$ is the coherence length, $l$ the electron mean free path, and $\delta$ a constant of order unity. For the case when $l$ is much less than $\xi_{0}(T)$, and as $\omega \rightarrow 0$, the relation is local: 


$$
\overrightarrow{\mathrm{j}}(\overrightarrow{\mathrm{r}}, \omega \rightarrow 0)=\frac{2 \delta l e^{2} N(0) v_{\boldsymbol{F}}}{3 \pi \hbar c} I(0,0, T) \overrightarrow{\mathrm{A}}(\overrightarrow{\mathrm{r}}) .
$$

For such low frequencies, $\omega \ll 2 \Delta_{0} / \hbar$, the electical conductivity is imaginary. $\sigma_{2}(\omega) / \sigma_{N}$ depends on frequency but is independent of wave number. In the weak-coupling theory calculated by Mattis and Bardeen ${ }^{14}$

$$
I(0,0, T)=-\pi^{2} \Delta_{0}(t) \tanh \left(\frac{\alpha}{4 t} \frac{\Delta_{0}(t)}{\Delta_{0}(0)}\right),
$$

where $\alpha=2 \Delta_{0}(0) / k_{B} T_{c}=3.52$ and $\Delta_{0}(t)$ is the BCS gap parameter.

In the strong-coupling case, Nam's ${ }^{7}$ Eqs. (4.34) and (4.35) give the expression for $I(0,0, T)$,

$$
I(0,0, T)=-\pi \lim _{\omega \rightarrow 0}\left(\omega \sigma_{2}\right),
$$

where

$$
\begin{aligned}
\sigma_{2}= & \frac{1}{\omega} \int_{\omega_{g}-\omega}^{\omega_{g}} g_{2}\left(\omega^{\prime}, \omega+\omega^{\prime}\right) \tanh \left[\frac{1}{2} \beta\left(\omega+\omega^{\prime}\right)\right] d \omega^{\prime} \\
& +\frac{1}{\omega} \int_{\omega_{g}}^{\infty}\left\{g_{2}\left(\omega^{\prime}, \omega+\omega^{\prime}\right) \tanh \left[\frac{1}{2} \beta\left(\omega+\omega^{\prime}\right)\right]\right. \\
& \left.+g_{2}\left(\omega+\omega^{\prime}, \omega^{\prime}\right) \tanh \left(\frac{1}{2} \beta \omega^{\prime}\right)\right\} d \omega^{\prime}
\end{aligned}
$$

and

$$
\begin{aligned}
g_{2}\left(\omega^{\prime}, \omega+\omega^{\prime}\right)= & \operatorname{Im}\left(\frac{\omega^{\prime}}{\left[\omega^{\prime 2}-\Delta^{2}\left(\omega^{\prime}\right)\right]^{1 / 2}}\right) \\
& \times \operatorname{Re}\left(\frac{\omega+\omega^{\prime}}{\left[\left(\omega+\omega^{\prime}\right)^{2}-\Delta^{2}\left(\omega+\omega^{\prime}\right)\right]^{1 / 2}}\right) \\
& +\operatorname{Im}\left(\frac{\Delta\left(\omega^{\prime}\right)}{\left[\omega^{\prime 2}-\Delta^{2}\left(\omega^{\prime}\right)\right]^{1 / 2}}\right) \\
& \times \operatorname{Re}\left(\frac{\Delta\left(\omega+\omega^{\prime}\right)}{\left[\left(\omega+\omega^{\prime}\right)^{2}-\Delta^{2}\left(\omega+\omega^{\prime}\right)\right]^{1 / 2}}\right) .
\end{aligned}
$$

B. Integration before limit approach

Harris has written a program ${ }^{13}$ to calculate $\sigma_{2}$ at several low frequencies for $T=0$, the results of which can be graphically extrapolated to $\omega=0$ to obtain $I(0,0,0)$ from Eq. (6). The information required for this procedure is the gap function $\Delta(\omega)=\Delta_{r}(\omega)+i \Delta_{i}(\omega)$ over the range of frequencies $\omega=0$ to a high-frequency cutoff (usually about 10 times $\bar{\omega}$, where $\bar{\omega}$ is the average phonon frequency). Such information is readily calculated during the inversion of tunneling data. This procedure can be used at higher temperatures, but then the temperature-dependent values of $\Delta(\omega)$ must be determined from solutions of the Eliashberg equations on the real frequency axis using tunneling derived $\alpha^{2}(\omega) F(\omega)$. A simpler procedure for the calculation of $I(0,0, T)$ involves the calculation of $\Delta\left(i \omega_{n}\right)$ on the imaginary axis as outlined in Sec. II D below.

We have used Harris' program to calculate $I(0,0,0)$ for $\mathrm{Pb},{ }^{15} \mathrm{~Pb}_{0.90} \mathrm{Bi}_{0.10},{ }^{15} a-\mathrm{Pb}_{0.45} \mathrm{Bi}_{0.55},{ }^{16}$ and $a-\mathrm{Bi},{ }^{17}$ with tunneling derived $\Delta(\omega)$ taken from the indicated references. Since $\Delta_{r}(\omega)$ and $\Delta_{i}(\omega)$ are derived only for $\omega \geq \omega_{g}$, whereas the integral contributes significantly for $0<\omega \leq \omega_{\mathrm{g}}$ also, we took $\Delta_{i}(\omega)=0$ in this range. This is exact at $T=0$ and a good approximation at low $T$. A first approximation to $\Delta_{r}(\omega)$ in this range is $\Delta_{r}(\omega)=\omega_{g}$, but this is not accurate because it gives zero slope to $\Delta_{r}(\omega)$. (See Sec. II C.) Better results were obtained by

\begin{tabular}{|c|c|c|c|c|c|}
\hline $\begin{array}{l}\text { Method of } \\
\text { calculation }\end{array}$ & $\mathrm{Pb}^{\mathrm{a}}$ & $\mathrm{Pb}_{0.90} \mathrm{Bi}_{0.10}{ }^{\mathrm{a}}$ & $a-\mathrm{Pb}_{0.45} \mathrm{Bi}_{0.55}^{\mathrm{b}}$ & $a-\mathrm{Bi}^{\mathrm{a}, \mathrm{d}}$ & $a-\mathrm{Sn}_{0.90} \mathrm{Cu}_{0.10} e^{e}$ \\
\hline $\begin{array}{l}\text { Integration } \\
\text { before limit }\end{array}$ & 1.63 & 1.82 & 1.64 & 1.37 & \\
\hline $\begin{array}{l}\text { Limit before } \\
\text { integration }\end{array}$ & 1.62 & 1.81 & 1.64 & 1.38 & \\
\hline Eliashberg & 1.65 & 1.83 & 1.69 & 1.42 & 1.59 \\
\hline Cauchy & 1.64 & 1.83 & 1.61 & 1.37 & \\
\hline $\begin{array}{l}\text { Sources of data } \\
{ }^{\mathbf{a}} \text { Reference } 1 \\
{ }^{\mathbf{b}} \text { Reference } 1 \\
{ }^{c} \text { Reference } 1\end{array}$ & $\omega)$ & $\left.{ }^{2}(\omega) F(\omega)\right]:$ & \multicolumn{2}{|c|}{$\begin{array}{l}{ }^{\mathrm{d}} \text { Reference } 20 . \\
{ }^{\mathrm{e}} \text { Reference } 21 .\end{array}$} & \\
\hline
\end{tabular}
using the Kramers-Kronig relation to compute values of $\Delta(\omega)$ in this range, thus giving $\Delta_{r}(\omega)$ a nonzero slope in this range.

The results of this calculation and others (to be discussed below) are compared in Table I.

TABLE I. Results of our different methods of calculating $I(0,0,0)$ for various superconductors. The values listed for Eliashberg and Cauchy were actually computed at $T=2.0^{\circ} \mathrm{K}$, but due to the flatness of the $I(0,0, T)$ vs $T$ curve at low $T$, they should be very close to the values at $T=0^{\circ} \mathrm{K}$. Values of $-I(0,0, T)$ below are in units of $10^{13} \mathrm{sec}^{-1}$. 


\section{Limit before integration approach}

Rather than performing both integrations of Eq. (7) to calculate $I(0,0, T)$, Kerchner and Ginsberg ${ }^{11}$ showed that for finite temperature the first integral vanishes as $\omega \rightarrow 0$, and hence one may write

$$
\begin{aligned}
I(0,0, T>0)=\pi \operatorname{Im} \int_{0}^{\infty} & \frac{\omega^{\prime 2}+\Delta^{2}\left(\omega^{\prime}\right)}{\omega^{\prime 2}-\Delta^{2}\left(\omega^{\prime}\right)} \\
& \times \tanh \left(\frac{1}{2} \beta \omega^{\prime}\right) d \omega^{\prime} .
\end{aligned}
$$

They stated that this expression is correct for $T$ $=0$ if one assumes that $\omega$ has a vanishingly small imaginary part. Calculations were presented for $I(0,0,0)$ for $\mathrm{Pb}$ and for $\mathrm{Pb}_{0.90} \mathrm{Bi}_{0.10}$ with the results being $-1.50 \times 10^{13} \mathrm{sec}^{-1}$ and $-1.61 \times 10^{13} \mathrm{sec}^{-1}$, respectively.

Lejeune and Naugle ${ }^{5}$ worked out the $T=0$ result starting from Eq. (8) and concluded that

$I(0,0,0)=-\pi^{2} \omega_{g}+\pi \operatorname{Im} \int_{\omega_{g}}^{\infty} \frac{\omega^{\prime 2}+\Delta^{2}\left(\omega^{\prime}\right)}{\omega^{\prime 2}-\Delta^{2}\left(\omega^{\prime}\right)} d \omega^{\prime}$.

They computed the value of $I(0,0,0)$ for $\mathrm{Pb}$ and got $-1.51 \times 10^{13} \mathrm{sec}^{-1}$. Their value for amorphous Bi was $-1.106 \times 10^{13} \mathrm{sec}^{-1}$.

Our calculations for $\mathrm{Pb}, \mathrm{Bi}$, and $\mathrm{Pb}_{0.90} \mathrm{Bi}_{0.10}$ using the other methods described in this paper gave the values of about $-1.63 \times 10^{13},-1.39 \times 10^{13}$, and $-1.83 \times 10^{13} \mathrm{sec}^{-1}$. To discover the reason for these discrepancies, we carefully reexamined the first integral in Eq. (7) in the limit $\omega \rightarrow 0$. It turns out that the slope of $\Delta_{r}(\omega)$ for $\omega \simeq \omega_{g}$ must be accounted for, and when this is done in taking the limit of Eq. (6), the first term on the right-hand side of Eq. (9) becomes

$$
-\pi^{2}\left(\frac{\omega_{g}}{1-\left(d \Delta_{r} / d \omega\right)_{\omega=\omega_{g}}}\right) \text {. }
$$

This is the same result as determined by Fulton and $\mathrm{McCumber}^{18}$ in their paper on the Josephson effect in strong-coupling superconductors. This provides a correction to Kerchner and Ginsberg's $T=0$ calculation and Eq. (14) of Lejeune and Naugle. With this correction, the values of $I(0,0,0)$ for $\mathrm{Pb}, \mathrm{Bi}$, and $\mathrm{Pb}_{0.90} \mathrm{Bi}_{0.10}$ were determined to be $-1.62 \times 10^{13},-1.38 \times 10^{13}$, and -1.81 $\times 10^{13} \mathrm{sec}^{-1}$, in excellent agreement with the results of our other calculations.

\section{Eliashberg approach}

According to $\mathrm{Nam}^{8} I(0,0, T)$ may also be expressed as

$$
I(0,0, T)=\frac{-4 \pi^{2} k T}{\hbar} \sum_{n \geqslant 0} \frac{\Delta_{n}^{2}}{\omega_{n}^{2}+\Delta_{n}^{2}},
$$

where $\Delta_{n} \equiv \Delta\left(i \omega_{n}\right)$ is the gap evaluated on the imaginary frequency axis at the frequency $i \omega_{n}$ $=i(2 n+1) C T\left(C=(\pi k / e) 10^{3}\right.$ for $\Delta_{n}$ and $\omega_{n}$ in units of $\mathrm{meV})$. To determine the set of $\Delta_{n}$ 's, the nonlinear Eliashberg equations must be solved ${ }^{19}$ :

$$
\begin{aligned}
& \tilde{\Delta}(n)=C T \sum_{m=-n}^{n-1}\left[\lambda\left(\omega_{n}-\omega_{m}\right)-\mu^{*}\right] \frac{\tilde{\Delta}(m)}{\left[\tilde{\omega}^{2}(m)+\tilde{\Delta}^{2}(m)\right]^{1 / 2}} \\
& \tilde{\omega}(n)=\omega_{n}+C T \sum_{m=-n}^{n-1} \lambda\left(\omega_{n}-\omega_{m}\right) \frac{\tilde{\omega}(m)}{\left[\tilde{\omega}^{2}(m)+\tilde{\Delta}^{2}(m)\right]^{1 / 2}},
\end{aligned}
$$

where $\tilde{\Delta}(n)=Z_{n} \Delta_{n}, \tilde{\omega}(n)=Z_{n} \omega_{n}$,

$$
\lambda\left(\omega_{n}-\omega_{m}\right)=2 \int_{0}^{\infty} \frac{\omega \alpha^{2} F(\omega)}{\omega^{2}+\left(\omega_{n}-\omega_{m}\right)^{2}} d \omega
$$

and $Z_{n} \equiv Z\left(i \omega_{n}\right)$ is the renormalization function on the imaginary frequency axis.

To solve these equations one must have the set of $\alpha^{2} F(\omega)$ for the particular superconductor under investigation [sources of $\alpha^{2} F(\omega)$ for the materials studied are indicated in Table I] along with a reasonable estimate of $\mu^{*}$ and a set of $N$ initial values for the gaps $\Delta_{n}$. Although the sums contain $2 N$ terms, the even nature of $\Delta_{n}$ and $Z_{n}$ (i.e., $\Delta_{n}=\Delta_{-n-1}$ ) can be exploited so that only $N$ different values of $\Delta_{n}$ and $Z_{n}$ need be considered. The maximum size of $n$ is determined by defining a cutoff frequency $\omega_{\max }=(2 N+1) C T$. We took $\omega_{\max }$ equal to ten times the maximum phonon frequency.

The equations were solved through an iteration and extrapolation procedure involving the set of $N \Delta_{n}$ 's. On each iteration a set of $\Delta_{n}$ 's (usually from the previous iteration) was put into the equations to generate a new set of $\Delta_{n}$ 's, the $\Delta_{n}^{\prime}$ 's. The quantity

$$
\lambda=\sum_{n}\left(\Delta_{n}-\Delta_{n}^{\prime}\right)^{2} / \sum_{n} \Delta_{n}^{2}
$$

was then computed. For a perfectly converged solution $\lambda=0$. Upon completing a calculation using only a direct iteration procedure, it was noticed that after the first few iterations, each of the $\Delta_{n}$ 's for the succeeding iterations was related in a nearly linear manner to the quantity $\lambda^{1 / 2}$ computed for each iteration. Hence an extrapolation procedure was set up. First a set of direct iterations (usually ten) was made. From that point, after every two direct iterations, the next set of $\Delta_{n}$ 's was determined by linearly extrapolating each of the $\Delta_{n}$ vs $\lambda^{1 / 2}$ curves to $\lambda^{1 / 2}=0$. Using this procedure, often only one extrapolation was necessary to reach the desired level of convergence. (Note that one $\Delta_{n}$ did not behave linearly. This occurred at the value of $n$ where the $\Delta_{n}$ 's changed sign. Since the magnitude of this one $\Delta_{n}$ was very small, the error introduced by using a linear extrapolation in this case was negligible.)

The iteration and extrapolation procedure for the 


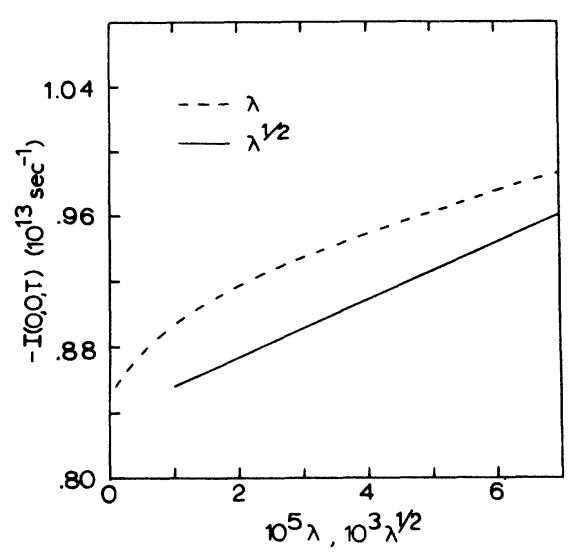

FIG. 1. Relationship between the electromagnetic response function $I(0,0, T)$ for $\mathrm{Pb}$ at $T=6.0^{\circ} \mathrm{K}$ as calculated by the Eliashberg method and the convergence test quantities $\lambda$ and $\lambda^{1 / 2}$ [see Eq. (13)].

$\Delta_{n}$ 's was continued until $\lambda<10^{-6}$ (or $\lambda^{1 / 2}<10^{-3}$ ). At this point of convergence, a second extrapolation was used to determine the value of $I(0,0, T)$ at $\lambda=0$. Figure 1 shows $I(0,0, T)$ as a function of the two quantities $\lambda$ and $\lambda^{1 / 2}$ for $\mathrm{Pb}$ at $T=6.0^{\circ} \mathrm{K}$. It can be seen from the figure that $I(0,0, T)$ exhibits a great deal of curvature as $\lambda \rightarrow 0$, making inaccurate any value of $I(0,0, T)$ obtained by linearly extrapolating this curve to $\lambda=0$ from points where $\lambda>10^{-5}$. The relationship between $I(0,0, T)$ and $\lambda^{1 / 2}$ is much more linear, thus permitting a reliable value of $I(0,0, T)$ to be obtained by extrapolating to $\lambda^{1 / 2}=0$. All of the Eliashberg values of $I(0,0, T)$ listed in this paper were obtained by such an extrapolation. By carrying the iteration procedure out to $\lambda^{1 / 2}<10^{-3}$ before extrapolating we found that generally the percentage difference between the extrapolated value of $I(0,0, T)$ at $\lambda^{1 / 2}=0$

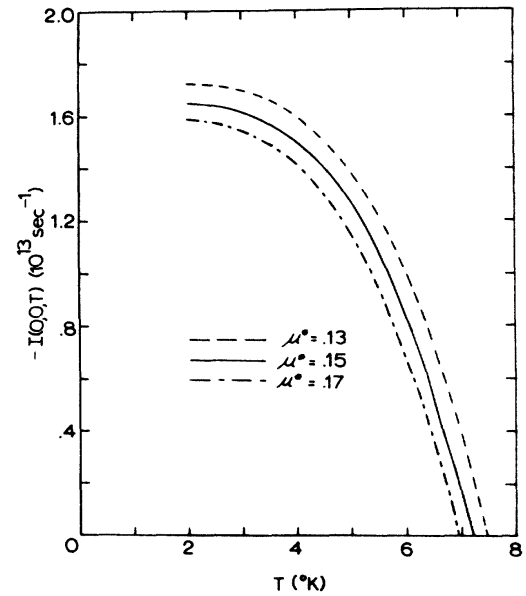

FIG. 2. $I(0,0, T)$ for $\mathrm{Pb}$ as a function of temperature for three values of $\mu^{*}$. and the value at $\lambda^{1 / 2} \leq 10^{-3}$ was on the order of $1 \%$.

It should be pointed out that solving the Eliashberg equations also yields values of the renormalization function $Z_{n}$. However, since our test of convergence was based only on the $\Delta_{n}$ 's, we cannot say to what degree that $Z_{n}$ 's are converged.

This method of calculating $I(0,0, T)$ has some distinct advantages. In terms of efficiency, the nonlinear coupled Eliashberg equations are much more readily solved in summation form than in integral form. ${ }^{22}$ Also results over a very wide temperature range can be obtained It is possible to get very close to $T_{c}\left(T_{c}-T \simeq 0.10^{\circ} \mathrm{K}\right)$ and still have the solution set converge rapidly. At low temperatures, however, the solution procedure is hampered by the large size of $N\left(\propto T^{-1}\right)$. Evaluating $I(0,0, T)$ at temperatures close to zero is not really necessary, however, since the $I(0,0, T)$ vs $T$ curve flattens out at $T \rightarrow 0$. This flattening can appear at reduced temperatures as high as 0.30 (see Fig. 2).

The temperature dependence of $I(0,0, T)$ for two superconductors is shown in Fig. 3. It can be seen

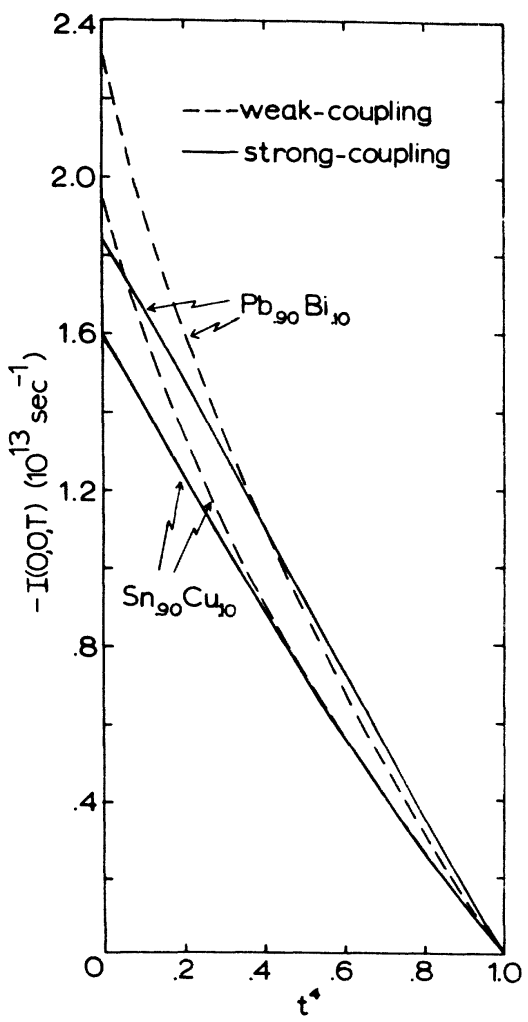

FIG. 3. $I(0,0, T)$ as a function of the fourth power of the reduced temperature for the alloys $\mathrm{Pb}_{0.90} \mathrm{Bi}_{0.10}$ and $\mathrm{Sn}_{0.90} \mathrm{Cu}_{0.10}$. The results of the strong-coupling theory are shown by the solid lines while the calculation from pure weak-coupling theory [using Eq. (15) with $\alpha=3.52$ ] are shown by the dashed lines. 
that $I(0,0, T)$ is nearly a linear function of the fourth power of the reduced temperature, $t^{4}$, which would be expected in the simple two-fluid model. Strong-coupling effects are noticeable in both the magnitude of $I(0,0,0)$ and the temperature dependence, $I(0,0, T) / I(0,0,0)$. As may be seen for both of the strong-coupling materials shown, $I(0,0,0)$ is appreciably reduced from the weakcoupling prediction, $-\pi^{2} \Delta_{0}(0) / \hbar$. The deviation of the temperature dependence from the weak-coupling prediction,

$$
\frac{I(0,0, T)}{I(0,0,0)}-\frac{\Delta_{0}(t)}{\Delta_{0}(0)} \tanh \frac{\alpha \Delta_{0}(t)}{4 t \Delta_{0}(0)},
$$

where $\alpha=3.52$ is shown as a function of $t$ in Fig. 4.

We conclude by noting that it was sometimes necessary to adjust $\mu^{*}$ to obtain the correct value for $T_{c}$. This should not be surprising, since it is well known that $\mu^{*}$ has a weak but sometimes significant dependence on the cutoff frequency on the real axis [here and throughout this paper, $\mu^{*}$ is the quantity $\mu^{*}\left(\omega_{\text {co }}\right)$ defined in Ref. 23], and here we are performing calculations on the imaginary axis. The corrections to $\mu^{*}$ have a significant effect on $I(0,0, T)$, as can be seen from Fig. 2 for $\mathrm{Pb}$. The adjustment of $\mu *$ to 0.15 (from the reported value of 0.13 ) gave the correct value for $T_{c}$ as well as a value of $I(0,0,0)$ in better agreement with the values obtained by the other methods. Similarly, for $\mathrm{Pb}_{0.45} \mathrm{Bi}_{0.55}$ it was necessary to adjust $\mu^{*}$ from 0.158 to 0.148 . Therefore, before employing real-frequency tunneling derived $\alpha^{2} F(\omega)$ and $\mu^{*}$ values for imaginary axis calculations, one must check that the correct value of $T_{c}$ is obtained.

\section{E. Cauchy approach}

As a check on the values of $\Delta_{n}$ and $Z_{n}$ obtained from the solution of the Eliashberg equations, a separate calculation using Cauchy's theorem was made. The gap functions on the imaginary axis can be determined from the real and imaginary parts of the real-frequency gap function $\Delta_{r}(\omega)$ and $\Delta_{i}(\omega)$ by

$$
\begin{aligned}
\Delta_{n} & =\frac{1}{2 \pi i} \int_{-\infty}^{\infty} \frac{\Delta_{r}(\omega)+i \Delta_{i}(\omega)}{\omega-i \omega_{n}} d \omega \\
& =\frac{1}{\pi} \int_{0}^{\infty} \frac{\omega \Delta_{i}(\omega)+\omega_{n} \Delta_{r}(\omega)}{\omega^{2}+\omega_{n}^{2}} d \omega .
\end{aligned}
$$

For Cauchy's theorem to be properly applied it is necessary that $\Delta(\omega)$ be analytic in the upper halfplane and that each term in the numerator of the integrand go to zero as $\omega \rightarrow \infty$. Therefore, the behavior of $\Delta_{r}(\omega)$ and $\Delta_{i}(\omega)$ must be examined to determine their limiting values as $\omega \rightarrow \infty$. There is no problem with $\Delta_{i}(\omega)$ as $\lim _{\omega \rightarrow \infty} \Delta_{i}(\omega)=0$. How-

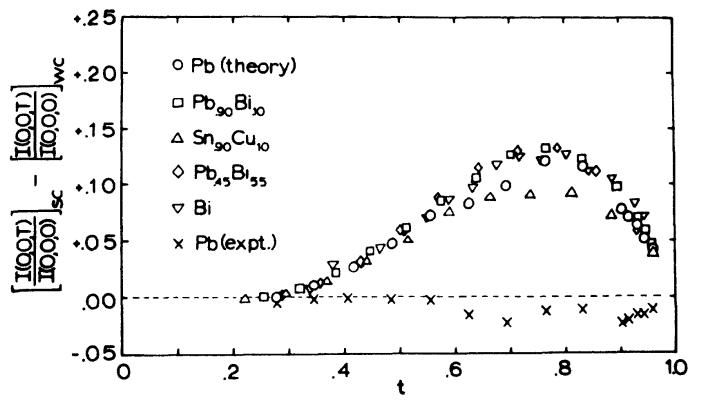

FIG. 4. Differences between calculated strongcoupling and weak-coupling values of $I(0,0, T) / I(0,0,0)$. All points except the $X$ 's represent theoretical calculations; the weak-coupling values were computed using Eq. (15) with $\alpha=3.52$. The $x$ 's denote the deviations between our strong-coupling calculations and the experimental results of Kerchner and Ginsberg for $\mathrm{Pb}$ $(\alpha=5.51)$.

ever, the value of $\Delta_{r}(\omega)$ is a small negative quantity at our upper limit of integration. This quantity must be subtracted from $\Delta_{r}(\omega)$ before integrating, and then added to the final result to get the correct $\Delta_{n}$. An entirely analogous procedure can be used to compute $Z_{n}$ from $Z_{r}(\omega)$ and $Z_{i}(\omega)$. In this case $\lim _{\omega \rightarrow \infty} Z_{r}(\omega)=1$ and $\lim _{\omega \rightarrow \infty} Z_{i}(\omega)=0$.

As previously noted, the tabular values of $\Delta_{r}(\omega)$, $\Delta_{i}(\omega), Z_{r}(\omega)$, and $Z_{i}(\omega)$ usually determined from inversion of tunneling data are only valid at low temperatures; hence the temperatures at which $\Delta_{n}$, $Z_{n}$, and $I(0,0, T)$ are calculated must also be low.

It was found that the values of $\Delta_{n}$ computed by the Eliashberg approach and those computed by the Cauchy method agree very well with each other. For $\mathrm{Pb}$ at $T=2{ }^{\circ} \mathrm{K}$, the percentage differences between the $\Delta_{n}$ 's was $0.9 \%$ for $\Delta_{0}, 1.3 \%$ for $\Delta_{5}$, and $3.8 \%$ for $\Delta_{90}$.

As before, once the $\Delta_{n}$ 's have been calculated, $I(0,0, T)$ can be determined from Nam's formula, Eq. (11). For low $T$, this approach has the advantages of being simple and fast.

\section{F. Comparison of calculated results}

Table I shows the values of $I(0,0, T)$ for five superconductors as determined by each of the methods above. For the "integration before limit" and "limit before integration" methods, the values of the response function at $T=0$ are shown. The values shown for the Eliashberg and Cauchy methods are for $T=2{ }^{\circ} \mathrm{K}$. This was necessary to avoid using a value of $N$ that was unreasonably large. However, due to the flatness of the $I(0,0, T)$ vs $T$ curve at low temperatures, comparisons between these values and those calculated for $T=0$ for the other methods are valid. It should be noted that the limit before integration results, which include the 
correction in Eq. (10), have a larger uncertainty than those of the other methods. This is due to the uncertainty present in the calculation of $\left(d \Delta_{r} /\right.$ $d \omega)_{\omega=\omega_{g}}$ from tabular values of $\Delta_{r}(\omega)$. In each case we computed $\left(d \Delta_{r} / d \omega\right)_{\omega=\omega_{g}}$ by averaging the quantities $\left[\Delta\left(\omega_{g}+\omega^{\prime}\right)-\Delta\left(\omega_{g}\right)\right] \%\left(\omega^{\prime}-\omega_{g}\right)$ and $\left[\Delta\left(\omega_{g}\right)-\Delta\left(\omega_{g}-\omega^{\prime \prime}\right)\right] /\left(\omega_{g}-\omega^{\prime \prime}\right)$, where $\omega^{\prime}, \omega^{\prime \prime} \leq 0.2$ meV. The agreement between the different methods of calculating the low-temperature values of the response function must be considered excellent. There is also good agreement between the results given in Table $I$ and the calculations of $I(0,0,0) /$ $I(0,0,0)_{\mathrm{MB}}$ reported by other researchers. ${ }^{10,12}$

The temperature dependence of $I(0,0, T)$ has also been calculated for $\mathrm{Pb}$ and $\mathrm{Pb}_{0.90} \mathrm{Bi}_{0.10}$ by Kerchner and Ginsberg. ${ }^{4,24}$ Except at $T=0$, their calculations are in good agreement with ours.

\section{COMPARISON WITH EXPERIMENT}

Various experiments have been performed which can provide a measure of $I(0,0, T)$. Pb has been the superconductor most frequently studied. Schwidtal and Finnegan ${ }^{6}$ measured the maximum tunneling supercurrent $I_{0}$ in $\mathrm{Pb}-\mathrm{Pb}$ Josephson junctions and found an average value of $I_{0} / I_{0 w}$ to be $0.784 \pm 0.006$ at $T=1.4^{\circ} \mathrm{K}$, where $I_{0 w}$ is the current calculated by weak-coupling theory. This ratio should be very close to $I(0,0,0) / I(0,0,0)_{M B}$, where $I(0,0,0)_{\mathrm{MB}}=-\pi^{2} \Delta_{0}(0) / \hbar$, since there is very little difference between the values of the gap at $1.4^{\circ} \mathrm{K}$ and at $0.0^{\circ} \mathrm{K}$. With our best calculated value of $I(0,0,0)=-1.63 \times 10^{13} \mathrm{sec}^{-1}$ and the value $\Delta_{0}(0)$ $=1.40 \mathrm{meV}$, the strong-coupling prediction is $I(0,0,0) / I(0,0,0)_{M B}=0.777$, in excellent agreement with experiment.

Measurements of the magnetic field attenuation in cylindrical thin $\mathrm{Pb}$ films were carried out by Kerchner and Ginsberg. ${ }^{4}$ They showed how the ratio of the applied field change to the attenuated field change can be expressed in terms of $I(0,0, T)$. Five $\mathrm{Pb}$ films were studied and the overall best value of $I(0,0,0)$ was reported to be $(-1.37 \pm 0.06)$ $\times 10^{13} \mathrm{sec}^{-1}$. However, from their Fig. 3, it appears that at least two of their five samples could yield values of $I(0,0,0)$ very close to $-1.63 \times 10^{13}$ $\mathrm{sec}^{-1}$. Ginsberg has requested that we point out that the theoretical values for $I(0,0,0)$, referred to in Ref. 4, and as well in Ref. 11 should be changed to those given in Table II in accordance with the correction mentioned in Sec. II C.

Kerchner and Ginsberg ${ }^{4}$ observed that the derived values of $I(0,0, T)$ from their measurements could be written in the same form as given by weak-coupling theory ${ }^{14}$

$$
I(0,0, T)=I(0,0,0) \frac{\Delta_{0}(t)}{\Delta_{0}(0)} \tanh \left(\frac{\alpha}{4 t} \frac{\Delta_{0}(t)}{\Delta_{0}(0)}\right),
$$

where $t$ is the reduced temperature, $\Delta_{0}(t) / \Delta_{0}(0)$ is the ratio of the energy gaps as determined by weak-coupling theory and tabulated by Muhlschlegel, ${ }^{25}$ and $\alpha$ is an adjustable parameter. The value of $\alpha$ which best fitted their data was $\alpha=5.5$ as compared to $\alpha=2 \Delta_{0}(0) / k_{B} T_{c}=3.52$ for the weakcoupling theory and $\alpha=4.51$ for the measured ratio of $2 \Delta_{0}(0) / k_{B} T_{c}$.

Lejeune and Naugle ${ }^{5}$ studied the kinetic inductance of thin $a-\mathrm{Bi}$ films and showed that the kinetic inductance is inversely proportional to $I(0,0, T)$. Since the kinetic inductance is very small, an absolute value of $I(0,0, T)$ could not be determined, but the temperature dependence could be observed. With the value of $I(0,0,0)$ computed from strong-

TABLE II. Comparison of strong-coupling calculations of $I(0,0, T)$ with experiment. The theoretical calculations have been fitted to the form of Eq. (14) to determine the parameter $\alpha_{\mathrm{sc}}$. The values $\alpha_{\mathrm{tum}}=2 \Delta_{0}(0) / k_{B} T_{c}$ are determined from the energy gap as measured in tunneling experiments. The values of $\alpha_{\text {exp }}$ is determined from a fit of experimental data to the form of Eq. (15). $I(0,0,0)_{\mathrm{sc}}$ and $I(0,0,0)_{\mathrm{exp}}$ are the calculated and experimental values, respectively.

\begin{tabular}{|c|c|c|c|c|c|}
\hline Material & $\alpha_{\mathbf{s c}}$ & $\alpha_{\text {tum }}$ & $\alpha_{\exp }$ & $\begin{array}{c}\text { Parameter } \\
-I(0,0,0)_{\mathrm{sc}} \\
\left(10^{13} \mathrm{sec}^{-1}\right)\end{array}$ & $\begin{array}{r}-I(0,0,0)_{\exp } \\
\left(10^{13} \mathrm{sec}^{-1}\right)\end{array}$ \\
\hline $\mathrm{Pb}$ & $4.95^{\mathrm{a}}$ & $4.51^{d}$ & $5.51^{b}$ & $1.63^{2}$ & $1.37^{\mathrm{b}}$ \\
\hline $\mathrm{Pb}_{0.90} \mathrm{Bi}_{0.10}$ & $5.05^{\mathrm{a}}$ & $4.55^{d}$ & ... & $1.83^{2}$ & $\ldots$ \\
\hline$a-B i$ & $4.86^{2}$ & $4.70^{\circ}$ & $2.48^{2, c}$ & $1.39^{2}$ & $\ldots$ \\
\hline$a-\mathrm{Pb}_{0.45} \mathrm{Bi}_{0.55}$ & $4.93^{2}$ & $4.80^{l}$ & $\cdots$ & $1.65^{2}$ & $\cdots$ \\
\hline$a-\mathrm{Sn}_{0.90} \mathrm{Cu}_{0.10}$ & $4.36^{\mathrm{a}}$ & $4.41^{\mathrm{g}}$ & $1.69^{2}$ & $1.59^{2}$ & $\cdots$ \\
\hline $\begin{array}{l}\text { Source of values: } \\
{ }^{2} \text { This paper. } \\
{ }^{b} \text { Reference } 4 . \\
{ }^{c} \text { Reference } 5 . \\
{ }^{d} \text { Reference } 15 .\end{array}$ & \multicolumn{5}{|c|}{$\begin{array}{l}{ }^{\mathbf{e}} \text { Reference } 17 . \\
{ }^{\mathrm{P}} \text { Reference } 16 . \\
{ }^{\mathbf{B}} \text { Reference } 21 .\end{array}$} \\
\hline
\end{tabular}


coupling theory the data can be fitted to the form of Eq. (15). In their calculation of $I(0,0,0)$ from Eq. (9), the correction reported in this paper was not included. Consequently, both the value of $I(0,0,0)$ and the value of $\alpha$ reported are incorrect. With the correct calculation of $I(0,0,0)=-1.37$ $\times 10^{13} \mathrm{sec}^{-1}$ for $a-\mathrm{Bi}$, the value of $\alpha$ which fits their data is $\alpha=2.48$ as compared to the measured gap ratio of 4.70 and weak-coupling value of 3.52. This new result leads to a change of scale in Lejeune and Naugle's Fig. 4 which shows the deviation of the experimental data from the prediction of Eq. (15). The values of $\left(I^{-1}\right)^{\prime}$ should be reduced by a factor of 0.804 . Further measurements of the kinetic inductance of amorphous films have been completed and the preliminary results for amorphous $\mathrm{Sn}_{0.90} \mathrm{Cu}_{0.10}$ are given in Table II. The experimental techniques are the same as those described by Lejeune and Naugle. ${ }^{5}$

Also included in Table II are the values of $\alpha_{s c}$ as determined from a fit of the strong-coupling calculations to Eq. (15), calculated strong-coupling values of $I(0,0,0)_{\mathrm{sc}}, \alpha_{\mathrm{tun}}=2 \Delta_{0}(0) / k_{B} T_{c} \mathrm{de}-$ termined from tunneling experiments, and $\alpha_{\text {exp }}$ and $I(0,0,0)_{\text {exp }}$ determined from fits of the experimental data to Eq. (15). It should be noted that the value of $\alpha_{\text {sc }}$ determined from the strong-coupling theory is within $10 \%$ of $\alpha_{\text {tun }}$ for all of the materials studied. As may be readily seen, the agreement between experiment and theory is reasonably good for $\mathrm{Pb}$; however, the agreement between theory and experiment is very poor for the amorphous metals where $\alpha_{\text {exp }}$ is appreciably smaller than $\alpha_{\text {sc }}$.

There are two possible explanations for the poor agreement between the theoretical calculations of the low-frequency electromagnetic response of amorphous metals and the available experimental data: either the theoretical calculations are inadequate for amorphous metals, or the interpretation of the kinetic inductance experiments is incorrect. For the crystalline strong-coupling superconductors, strong-coupling calculations of a wide variety of properties using tunneling derived $\alpha^{2}(\omega) F(\omega)$ are in quantitative agreement with experiment ${ }^{26}$ as in our calculation of $I(0,0, T)$ for $\mathrm{Pb}$. On the other hand, although there are numerous calculations of strong-coupling effects in amorphous superconductors, there are very few experiments which can test these calculations quantitatively. Inadequacies in the theoretical calculations could arise from the sensitivity to the anomalously large contribution to $\alpha^{2}(\omega) F(\omega)$ at low frequency which is generally observed for amorphous metals and which also is difficult to determine precisely from tunneling measurements. Perhaps there is an inadequacy in the approximations of strong-coupling theory which is accentuated for materials with $\alpha^{2}(\omega) F(\omega)$ characteristic of amorphous metals. The experimental difficulties with kinetic inductance measurements and possible sources of error in their interpretation are discussed in more detail below.

The principal measurements on amorphous superconductors which could be compared with strong-coupling calculations are the perpendicular critical field measurements by Bergmann, ${ }^{27}$ the infrared transmission measurements by Harris and Ginsberg, ${ }^{1}$ and the microwave surface experiments by Reichert and Hasse. ${ }^{28}$ Bergmann's measurements showed a low-temperature critical field for amorphous $\mathrm{Pb}_{0.75} \mathrm{Bi}_{0.25}$ that was larger than that expected from the BCS temperature dependence, but that was in very good agreement with strongcoupling calculation ${ }^{29}$ using tunneling derived $\alpha^{2}(\omega) F(\omega)$. Harris and Ginsberg compared their experiments with strong-coupling calculations based on Nam's theory ${ }^{7,8}$ of the electromagnetic response in strong-coupling superconductors (as were the calculations in this paper). They reported good agreement between theory and experiments; however, it was necessary to shift the frequency scale of the calculations to obtain this agreement. This was apparently forced by the fact that the films in their experiment were very thin and had gaps which differed from those of the thicker films. This complication and the fact that their lowest measured frequencies were still above $\frac{1}{2} \Delta_{0}$ prevented extrapolation of their cata to $\omega=0$ to determine $I(0,0,0)$ for comparison with our calculations. Unfortunately, the measurements of Reichert and Hasse $^{28}$ were not compared to Nam's theory and we are unable to compare quantitatively their data as presented to the theory.

There are several experimental artifacts which could complicate the interpretation of kinetic inductance measurements and invalidate the direct relation of the data to $I(0,0, T)$. Possible problems are (a) nonequilibrium behavior due to the power level, (b) contribution to the kinetic inductance from magnetic flux lines and (c) a nonuniform current distribution.

In the particular experiments the power was determined by choice of the tunnel diode oscillator, a Bd-5, and could not be adjusted during the experiment. We are aware of no calculations of the effect of a nonequilibrium distribution nor experiments to study the influence of the power level on kinetic inductance measurements. Although no appreciable shift in $T_{c}$ as extrapolated from kinetic inductance measurements was observed, this possibility cannot be ruled out. The low-frequency (about $10 \mathrm{MHz}$ ) of the experiments, however, does exclude finite frequency effects as an explanation.

Little $^{30}$ has reported an influence of a perpen- 
dicular magnetic field on the kinetic inductance of thin superconducting films. For the experiments of Lejeune and Naugle ${ }^{5}$ the films were surrounded by a $\mu$-metal shield which would appreciably reduce the earth's ambient field orders of magnitude below those used in Little's experiments. It is doubtful that such small fields could produce the disagreement observed between theory and experiment.

The discrepancy between theory and experiment could be readily explained if the current distribution in the amorphous films of the kinetic inductance experiments were sufficiently nonuniform to give a value of $\left\langle j^{2}\right\rangle /\langle j\rangle^{2}=2.4$ for $a-\mathrm{Bi}$ and $\left\langle j^{2}\right\rangle /\langle j\rangle^{2}$ $=3.3$ for $a-\mathrm{Sn}_{0.90} \mathrm{Cu}_{0.10^{\circ}}$. Baker et al ${ }^{31}$ have studied the kinetic inductance of superconducting films as a function of cross-sectional area and concluded that the current distribution in these films, which are condensed onto a superconducting $\mathrm{Nb}$ shield plane, is essentially uniform. Such a large variation in current density could be consistent with their measurements only if the data for the film with the smallest cross section $\left(3 \times 10^{-8} \mathrm{~cm}^{2}\right)$ had considerably more error than estimated. This large a variation is also inconsistent with critical current measurements using single and double superconducting shield planes ${ }^{32}$ and the qualitative argument that the current distribution should be uniform whenever the kinetic inductance dominates the magnetic inductance of the film on the shield plane. For these experiments the kinetic inductance is estimated to be an order of magnitude larger than the magnetic inductance of the film.

The calculations for the kinetic inductance experiments were done with $\delta$ set equal to unity in Eq. (4). By decreasing $\delta$ the experimental results can be brought into better agreement with theory. In the case of $a-\mathrm{Bi} \delta$ would have to be 0.42 to account for all of the discrepancy. As this figure is only about half of the assumed value ${ }^{33}$ we do not believe that changing $\delta$ can satisfactorily account for the difference between experiment and theory.

At this time we cannot resolve the discrepancy between theory and experiment. This discrepancy can most readily be resolved, and the theory be quantitatively tested, by experiments on the penetration depth for a thin superconducting cylinder made from an amorphous superconductor analogous to the experiments by Kerchner and Ginsberg ${ }^{4}$ on crystalline $\mathrm{Pb}$ cylinders. The interpretation to provide an absolute measurement of $I(0,0, T)$ will be simplified by the local relation between $\vec{j}$ and $\vec{A}$ for amorphous metals.

\section{SUMMARY AND CONCLUSIONS}

We have shown how the electromagnetic response function $I(0,0, T)$ may be calculated by four dif- ferent techniques: (1) a direct calculation by Harris of $\sigma_{2}$ before taking $\lim \omega \rightarrow 0$, (2) a calclation by Kerchner and Ginsberg of $\sigma_{2}$ after taking $\lim \omega \rightarrow 0$, (3) a solution of the nonlinear Eliashberg equations on the imaginary axis, (4) an application of Cauchy's theorem. Of these techniques only the solution of the Eliashberg equations using the $\alpha^{2}(\omega) F(\omega)$ function is suited for calculation of $I(0,0, T)$ over a large temperature range. A technique for obtaining rapid convergence of the solution set $\Delta_{n}$ has been discussed which provides a convenient, fast, and inexpensive method for computation of the many values of $I(0,0, T)$ needed. A correction to the Kerchner and Ginsberg ${ }^{4}$ calculation for $I(0,0,0)$ was found which, when applied, produced a high degree of consistency among the results obtained by each of the methods.

The results of the calculations are in good agreement with experiment for crystalline $\mathrm{Pb}$. The agreement between the calculations and the temperature dependence inferred from kinetic inductance measurements with amorphous films ${ }^{5}$ is very poor. Since the results of strong-coupling calculations for $H_{c 2}$ of $a-\mathrm{Pb}_{0.75} \mathrm{Bi}_{0.25}$ films are in good agreement with experiment, ${ }^{27}$ we suspect that the discrepancy lies in the theory of the electromagnetic response, its extension to low frequencies, or in the interpretation of the kinetic inductance data. Because of the variation of the energy gap for the very thin amorphous films used in infrared experiments, ${ }^{1}$ their usefulness in providing a quantitative test of the theory is restricted. The discrepancy in the temperature dependence of $I(0,0, T)$, as inferred from the kinetic inductance measurements when compared to those calculated from strong-coupling theory, can be explained if it is assumed that the current flows through less than $50 \%$ of the film in the kinetic inductance experiments. Such an explanation, however, is inconsistent with other experiments.

The most definitive way to resolve the present disagreement between theory and experiment would be to measure the field penetration depth for amorphous metal cylinders, such as was done in the experiments of Kerchner and Ginsberg. ${ }^{4}$ Although the fabrication of such cylinders would present more serious experimental problems than for those of crystalline metals, the analysis of the data would be greatly simplified by the use of local electrodynamics.

\section{ACKNOWLEDGMENTS}

The authors wish to acknowledge receipt of tabulated values of tunneling derived $\alpha^{2}(\omega) F(\omega)$ from N. Barth, R. Dynes, R. Harris, and J. Leslie and tabulated data from D. Ginsberg, R. Harris, 
and H. Kerchner. R. Harris has been particularly gracious in sending his programs for the calculation of $\sigma_{2}(\omega)$. Several of us have benefited from very useful conversations with $P$. Allen, R. Dynes,
D. Ginsberg, R. Harris, D. Rainer, J. Serene, and J. Swihart. J. Serene and H. Vidberg have kindly sent a preprint of their work on related topics.
*Work supported by the NSF.

$\dagger$ Present address: Texas Instruments, Richardson, Tex.

${ }^{1}$ R. E. Harris and D. M. Ginsberg, Phys. Rev. $\underline{188,} 737$ (1969).

${ }^{2}$ L. H. Palmer and M. Tinkham, Phys. Rev. 165, 588 (1968).

${ }^{3}$ S. L. Lehoczky and C. V. Briscoe, Phys. Rev. B $\underline{4}$, 3938 (1971).

${ }^{4}$ H. R. Kerchner and D. M. Ginsberg, Phys. Rev. B 10, 1916 (1974).

${ }^{5}$ J. D. Lejeune and D. G. Naugle, J. Low Temp. Phys. 22, 425 (1976).

${ }^{6}$ K. Schwidtal and R. D. Finnegan, Phys. Rev. B 2, 148 (1970).

${ }^{7}$ S. B. Nam, Phys. Rev. 156, 470 (1967).

${ }^{8}$ S. B. Nam, Phys. Rev. $\underline{156}, 487$ (1967).

${ }^{9} \mathrm{~J}$. C. Swihart and W. Shaw, in Superconductivity, edited by F. Chilton (North-Holland, Amsterdam, 1971), p. 678.

${ }^{10}$ J. P. Carbotte and P. Vashishta, Can. J. Phys. 49 , 1493 (1971).

${ }^{11}$ H. R. Kerchner and D. M. Ginsberg, Phys. Rev. B $\underline{8}$, 3190 (1973).

${ }^{12}$ D. M. Ginsberg, R. E. Harris, and R. C. Dynes, Phys. Rev. B 14, 990 (1976).

${ }^{13}$ R. E. Harris, Tech. Report No. 180, Dept. of Physics, University of nlinois, Urbana, nlinois, 1969 (unpublished).

${ }^{14}$ D. C. Mattis and J. Bardeen, Phys. Rev. 111, 412 (1958).

${ }^{15}$ J. M. Rowell, W. L. McMillan, and R. C. Dynes (unpublished).

${ }^{16}$ R. C. Dynes (private communication).

${ }^{17}$ R. E. Harris (private communication)

${ }^{18}$ T. A. Fulton and D. E. McCumber, Phys. Rev. $\underline{175}$, 585 (1968)

${ }^{19}$ D. Rainer and G. Bergmann, J. Low Temp. Phys. 14, 501 (1974).

${ }^{20} \mathrm{~J}$. D. Leslie, J. T. Chen, and T. T. Chen, Can. J. Phys. 48, 2783 (1970).

${ }^{21} \mathrm{~K}$. Knorr and N. Barth, Solid State Commun. $\underline{8}, 1085$ (1970).

${ }^{22}$ P. Vashishta and J. P. Carbotte, Phys. Rev. B $\underline{7}, 1874$ (1973).

${ }^{23}$ P. B. Allen and R. C. Dynes, Phys. Rev. B 12, 905 (1975).

${ }^{24} \mathrm{H}$. R. Kerchner, dissertation (University of mlinois, 1974) (unpublished).

${ }^{25}$ B. Mühlschlegel, Z. Phys. 155, 313 (1959).

${ }^{26}$ See, D. J. Scalapino, in Superconductivity, edited by R. D. Parks (Dekker, New York, 1969), Vol. 2, p. 449.

${ }^{27}$ G. Bergmann, Phys. Rev. B 7, 4850 (1973).

${ }^{28} \mathrm{~V}$. Reichert and J. Hasse, Z. Phys. 254, 10 (1972).

${ }^{29}$ D. Rainer, G. Bergmann and U. Eckhardt, Phys. Rev. B 8, 5324 (1973).

${ }^{30}$ W. Little, Proceedings of the Symposium on Superconducting Devices (University of Virginia, Charlottesville, 1967), paper 3-1 (unpublished).

${ }^{31} \mathrm{~J}$. W. Baker, J. D. Lejeune, and D. G. Naugle, J. Appl. Phys. 45, 5043 (1974).

${ }^{32}$ H. H. Edwards and V. L. Newhouse, J. Appl. Phys. 33, 868 (1962).

${ }^{33}$ A. B. Pippard, Proc. R. Soc. Lond. A 216, 547 (1953). 